\title{
Therapeutic Potential of Saffron Crocus (Crocus sativus L.)
}

\author{
Zeliha Selamoglu ${ }^{1}$, Senay Ozgen ${ }^{2 *}$ \\ ${ }^{I}$ Department of Biotechnology, Faculty of Arts and Science, Omer Halisdemir University, 51240 Niğde, Turkey \\ ${ }^{2}$ Department of Plant Productions and Technologies, Faculty of Agricultural Sciences and Technologies, Omer Halisdemir University,
} 51240 Niğde, Turkey

A R T I C LE INFO

\section{Article history:}

Received 03 November 2016

Accepted 30 November 2016

Available online, ISSN: 2148-127X

Keywords:

Biological activity

Folk medicine

Medicinal plants

Crocetin

Crocin \begin{abstract}
A B S T R A C T
Herbal plants are using for folk medicine since immemorial times. Hippocrates who is the father of medicine, refer to 400 medicinal plants and advised "let food be your medicine and let medicine be your food"'. Most of plants are using today for medicine. It is still acceptable today that prevention is more important than treatment. Saffron was utilized in folk remedy against scarlet fever, smallpox, colds, asthma, eye and heart diseases, tumours, and cancer.
\end{abstract}

\footnotetext{
${ }^{*}$ Corresponding Author:

E-mail: senayugur01@gmail.com
}

\section{Introduction}

Saffron is one of the most beneficial plants in the world from the member of Iridaceae family. It is known as saffron. Scientific classification of saffron is as follows: Kingdom: Plantae, Division: Magnoliophyta, Class: Liliopsida, Order: Asparagales, Family: Iridaceae, Genus: Crocus, Species: C. sativus L (Hagh-Nazari and Keifi, 2007). This family has about 80 species in the world and 32 of them are existing in Turkey. (Davis et al., 1988) reported that 18 species are endemic for flora of Turkey. One of them is the crocus species that are in the $30^{\circ}$ and $40^{\circ}$ North latitude. Flowering period of Crocus sativus are 2 or 3 weeks in October and November. Harvesting and cultivating to saffron is too hard. For this reasons saffron is one of the most expensive spice in the world (Hagh-Nazari and Keifi, 2007).

\section{History of Crocus Sativus and Importance in Folk Medicine}

The spice and the plant are usually calling the same name 'saffron'. Saffron has a long story to using in many dishes. Some archaeological and historical researches represent that domestication of saffron dates back to 2000-1500 years BC (Sharafzadeh, 2012). Turkey's Flora is very rich because of its geological structure, geological position and climate characters (Özdemir, 2006). Turkey's biogeography is very suitable for growing plants. Cultivating to saffron plant was very important in the period of Ottoman Empire. That time most of saffron was exporting other countries. But, saffron production has been decreased past from today. Saffron's cultivating is in small area of Safranbolu province (Arslan et al., 2007). Some reports show that the first product of saffron was in Turkey about $13^{\text {th }}-14^{\text {th }}$ centuries AD. Safranbolu province got its name from saffron. The city is the main saffron producer in Turkey (Mousavi and Bathaie, 2011). United National Educational Scientific and Cultural Organisation (UNESCO) were chosen Safronbolu, World Heritage City, in 1994 because of well-preserved Ottoman Houses and architecture (Caiola and Canini, 2010). Saffron is cultivated at least 3500 years in too many countries such as Iran, India, Pakistan, Greece, Spain, Italy, Turkey, France, Switzerland, Israel, Pakistan, Azerbaijan, China, Egypt, United Arab Emirates, Japan, Afghanistan, Iraq and recently Australia. Today Iran is one of the most important producer (Mousavi and Bathaie, 2011; Rahimi et al., 2013).

\section{Ethnomedical Importance of Saffron}

The research's showed that Assyrians and Babylonians used saffron to treat dyspnea, problems of head, menstruation, delivery and painful urination. The oldest document of saffron's medical use is in Assurbanipal library; in inscriptions dated back to $12^{\text {th }}$ century BC (Mousavi and Bathaie, 2011). In Ayurveda, 
saffron is used to treat chronic diseases such as asthma and arthritis. It is also using to treat colds and coughs. Ayurvedic medicines containing saffron are used to treat acne and several skin illnesses. Ancient texts showed that the herb's useful as an aphrodisiac, so, it is stimulating reproductive system. It is largely used as a folk medicine in India.

The stigma of this plant is also a well-known traditional Chinese medicine (Bhandari, 2015). In ancient Greece Saffron was a royal colorant and was utilized as a perfume in saloons, courts, theatres and bathrooms. Another use of saffron was as a herbal cure Hippocrates $\left(5-4^{\text {th }}\right.$ century BC), Erasistratus $\left(4-3^{\text {rd }}\right.$ century BC), Diokles $\left(3^{\text {rd }}\right.$ century BC) and Discorides $\left(1^{\text {st }}\right.$ century AD) utilized saffron for medical intentions like treating eye diseases (painful eye, corneal disease and cataract, purulent eye infection), earache, tooth-ache, ulcers (skin, mouth, genitalia) and erysipelas; they believed that it has styptic and sedative features (Mousavi and Bathaie, 2011).

\section{Saffron Bioactive Compounds}

Saffron's length is between the 10 to $30 \mathrm{~cm}$. Compact and globular corms calibre of $5 \mathrm{~cm}$ and established in the substructure. Every corm has 6 to 8 leaves. Outside of the single ovule ovary in the centre of the flower grows a thin style of a light yellow colour which ends in a triple stigma of 2-3 cm length and bright orange-red colour. It is the dried stigmas of saffron. The spice of saffron includes several chemical substances like carbohydrates, minerals, mucilage, vitamins (especially riboflavin and thiamin) and pigments including crocin, anthocyanin, carotene, lycopene, and zigzantin. There is also an aromatic essence turpenic (Safranal), and picrocrocin which give saffron its spesific taste. Saffron which has triploid 24 chromosomes is infertile (Moghadam et al., 2013; Singla and Varadaraj, 2011). Vegetative propagation is utilized for saffron propagation because of its triploid choromosomes (Arslan et al., 2007). Saffron is symbolized by its long red stigmas. This stigma's produce major amounts of carotenoids (Ahrazem et al., 2010). The stigma's have a fascinating and distinctive color, flavor and smell. Some chemical compounds responsible for its features:

Color: A main colouring pigment is crocin.

Smell: The principal aroma factor in saffron is safranal (Figure 1).

Flavor: The spesific "bitter" flavour is the glycoside picrocrocin (Abdullaev, 2007).

Crocins, picrocrocin and safranal is the main ingredient of saffron. Crocin's function is colouring. Picrocrocin and safranal's function is bitter taste of safron and its aroma. Picrocrocin $\left(\mathrm{C}_{16} \mathrm{H}_{26} \mathrm{O}_{7}\right)$ is responsible the major bitter taste. Saffron's aroma caused from essential oil and its major component is the terpene aldehyde, safranal. Crocins dissolve simply in water to supply an orange-red solution. This is the reason for using as a food colorant (Rahimi et al., 2013). Crocin is a natural carotenoid and is also found in Gardenia Jasmenoides (Ahmad Dar et al., 2012).

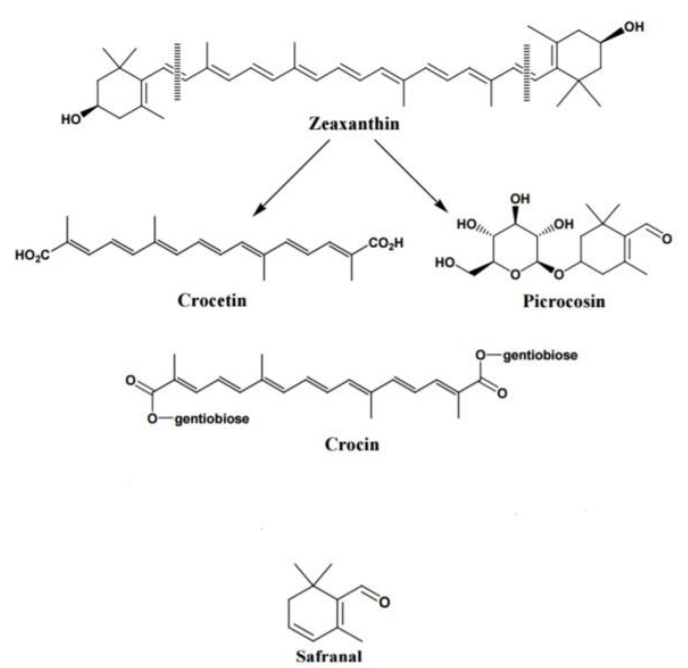

Figure 1 The structure of safranal (Leffingwell, 2002)

\section{Biological Activities of Saffron}

Various pharmacological activities of saffron have been investigated. Saffron and it's the most important component (crocin), have many therapeutic features such as antitumor, antioxidant, anxiolytic, neuronal protective, anti-ischemic and protective against DNA damage activities (Mohajeri et al., 2010). Saffron and its crocins are potential anti-cancer and also a hypolepidemic and antioxidant agent, protects brain against excessive oxidative stress, protective on ischemic heart, useful in alleviation of cognitive deficits, possesses significant antiproliferation effects on human prostate and colorectal cancer cells, aphrodisiac activity, immunomodulatory, growth inhibition of dalton's lymphoma, beneficial in diabetic neuropathy treatment, effects glucose uptake and insulin sensitivity and too many other activities. Another component of saffron, crocetin, enhances alveolar oxygen transport and raising pulmonary oxygenation, obstructs skin tumour promotion and maintains against oxidative damages, have cardiovascular protective effects, improves acetylcholine-induced vascular relaxation in hypertension, acts as potent anti-tumour agent, inhibits vascular endothelial growth factor-induced angiogenesis, antithrombotic effects (Agnihotri and Aperito, 2015).

Crocetin has many beneficial effects but on the contrary some researches showed that crocetin can be a teratogen. The high dose of crocetin caused abortion and it can be toxic (Mohajeri et al., 2007). Crocin has many biologic activities even so crocin $(200 \mathrm{mg} / \mathrm{kg}$, ip.) did not show anticonvulsant activity. The meaning of this is crocin must be a good anti-depressant (Ahmad Dar et al., 2012).

Crocin showed various pharmacological effects on the nervous system such as antianxiety activity, antidepressant effects, aphrodisiac properties, learning and memory-enhancement (Alavizadeh and Hosseinzadeh, 2014). Modaghegha et al. (2008) studied the effects of saffron tablets on hematological and biochemical parameters. Saffron stigma tablets were appraised for short-term safety and tolerability in healthy 
adult volunteers. 3 groups are generated. Each group has 10 volunteers (5 males and 5 females). Group I was placebo, meanwhile, groups 2 and 3 received 200 and 400 mg saffron tablets, respectively, for 7 days. Volunteers' hematological, biochemical and electrocardiographic parameters were measured pre and post treatment. Clinical findings showed no appearance changes in all volunteers after intervention. Higher dose of saffron (400 $\mathrm{mg}$ ) remarkable reduced standing systolic blood pressure and mean arterial pressures. Saffron reduced slightly some hematological parameters such as red blood cells, hemoglobin, hematocrit and platelets. Saffron improved sodium, blood urea nitrogen and creatinine amount (Modaghegha et al., 2008).

\section{Antiinflammatuar Effect of Saffron}

Antiinflamatuar effect of saffranal is investigated by Tamaddonfard et al. (2013). The research showed that crocin and safranal showed antiinflammatory and antinociceptive effects in carrageenan model of inflammation and inflammatory pain. Crocin and safranal anti-inflammatory and antinociceptive effects were the same as those of diclofenac, as a reference drug (Tamaddonfard et al., 2013). There are many studies that show the neuroprotective activities of crocin and crocetin in experimental models of brain disorders, for instantce cerebral ischemia, memory impairment and Parkinson's disease (Zhang et al., 1994; Zheng et al., 2007; Ahmad et al., 2005). In a hemorrhagic sshock model, crocetin suppressed mRNA expression of tumour necrosis factor$\alpha$, interleukin-1 $\beta$ and iNOSin her liver and raised the survival (Yang et al., 2006). Also, crocin raises intracellular glutathione levels, so that prohibits cell death in serum-deprived and hypoxic PC12 cells which is the cell culture model for brain ischemia (Ochiai et al., 2007). It has been shown that crocin protects the cerebral microvessels from high oxidative stress via decreasing the activities of superoxide dismutase and glutathione peroxidase (Zeng et al., 2007). Nam et al. (2010) demonstrated that one of the mechanisms of the inhibitory effects of the crocin and crocetin in activated microglia was the repression of the NF-KB transcriptional activity.

\section{Anti-Hyperglycemic Effect of Saffron}

Kianbakht and Hajiaghaee (2011) studied about saffron, crocin and safranal effects on the blood levels in diabetic rats. Their results showed that saffron may have anti-hyperglycemic and blood insulin level raising effects without hepatic and renal toxicities in the alloxan-diabetic rats. Crocetin was able to increase insulin sensitivity and ameliorated abnormalities correlated to insulin resistance, such as, impaired glucose tolerance, hypertension due to high-fructose diet and dexamethasone injection in rats (Chen et al., 2008; Xi et al., 2005). Also, crocetin reduced the palmitate-induced insulin sensitivity in the rat adipocytes ( $\mathrm{Xi}$ et al., 2007). Moreover, crocetin is enhanced the insulin resistance stimulated by high-fat diet in rats (Sheng et al., 2008). Furthermore, crocetin is blocked adhesion of leukocytes to the bovine aortic endothelial cells (BEC) stimulated by advanced glycation end products (AGEs) and AGEs-induced BEC apoptosis probably via its antioxidant activity, thus, researchers reported that crocetin may hinder diabetes-related vasvular complications (Xiang et al., 2006a; Xiang et al., 2006b). It has been proven that extraction of saffron improved the insulin level in alloxan-diabetic and nondiabetic rats and caused renewal of $\beta$-cells in alloxandiabetic rats (Mohajeri et al., 2009; Arasteh et al., 2010). As a result, all these effects may cause due to crocin, crocetin and safranal in saffron.

\section{Effect of Saffron on Central Nervous System}

Crocin has a special, protective effect on ethanolinduced impairment of learning and memory (Singla and Varadaraj, 2011). Sahraeil et al. (2012) examined that effects of saffrons ethanolic extract and it's the most important constituent crocin on chronic stress induced Wistar rats. Their results report that saffron ethanolic extract and crocin might interact with hormonal, metabolic and behavioural changes induced by electric shock stress in rats (Sahraeil et al., 2012). Alzheimer is a neurodegenerative disorder symbolized by the formation of extracellular amyloid $\beta$-peptide $(\mathrm{A} \beta)$ fibrils and intracellular neurofibrillary messes. It has been investigated that apoptotic cell death occurs in the brains of Alzheimer's patients. The study showed that the methanol and water extract of C.sativus stigmas inhibited $\mathrm{A} \beta$ fibrillogenesis in a concentration (Singla and Varadaraj, 2011; Alavizadeh and Hosseinzadeh, 2014). Saffron has effective on cerebral ischemia. The cumulative findings support the involvement of reactive oxygen species (ROS) in cerebral ischemia- induced neural cell damage. In fact, ROS are accountable for generating nitric oxide (NO) and malondialdehyde (MDA) and reducing activities of superoxide dismutase (SOD) and glutathione peroxidase (GSH). Antioxidants as $\alpha$-tocopherol, $\beta$-carotene, ascorbic acid and more, are considered promising for neuroprotection due to their potential ROS prohibit abilities. The studies showed that treatment of PC-12 cells with crocin inhibited cell membrane lipid peroxidation and restored intracellular SOD activity even more efficacious than a-tocopherol at the same concentration (Alavizadeh and Hosseinzadeh, 2014). Hosseinzadeh et al. (2012) studied about saffron and it's the major component crocin's effect on recognition and spatial memory after chronic cerebral hypoperfusion in Wistar rats. Their study commits that saffron extract and crocin improve spatial cognitive abilities following chronic cerebral hypoperfusion and the effect might be associated to the antioxidant effects of these compounds (Hosseinzadeh et al., 2012). It has been reported that saffron and its components possess antidepressant and anxiolytic effects (Alavizadeh and Hosseinzadeh, 2014). 


\section{Antioxidant Effect of Saffron}

Reactive oxygen species (ROS) cause oxidative damage in cell membranes and it is associated with many pathological conditions. Antioxidant therapy is well known method to protecting from ROS. Saffron has been advised for its radical scavenging feature that inhibits lipid peroxidation (Premkumbar and Ramesh, 2010). The crocin bleaching assay was also developed according to this important property of crocin as a basic element which is responsible for the antioxidant activity of saffron. The chemo preventive quality of saffron via modulation of antioxidants, lipid peroxidation, and detoxification systems was also suggested. Masmoul et al. (2013) report that saffron's antioxidant-rich components can be modulate obesity and associated disorders. They might be useful controlling, managing and preventing disorders associated with overweight and obesity (Mashmoul et al., 2013). There is a study to determine polyphenol amount in Lebanese saffron and the antioxidant effect from different extracts of this (Makhlouf et al., 2011). Their study showed that Crocus sativus, grown in Lebanon, includes a significant amount of polyphenols and it has a strong antioxidant effect against the free radicals. This plant supplies prevention for the organs free radical depended on damages. Even so, these effects depend on dose. Furthermore, it decreases the level of lipid peroxidation that might cause several problems and maintain the activity of one of the important antioxidant enzymes, the superoxide dismutase (Makhlouf et al., 2011).

\section{Cardiovascular Effects of Saffron}

Antioxidants in saffron tea can decrease the risk of cardiovascular diseases. The flavonoids, especially lycopene, found in saffron can supply added prevention. Crocetin implicitly helps to decrease cholesterol level in the blood and severity of atherosclerosis, thus decreasing the chances of heart attacks. Saffron helps to decrease the risk of heart disorders by reinforcement the blood circulatory system. Saffron has minerals such as thiamin and riboflavin. Saffron promotes a healthy heart and prevents various cardiac disorders (Kamalipour and Akhondzadeh, 2011). Atherogenic dyslipidemia is a main risk factor for cardiovascular diseases in diabetic patients. It is qualified with a raised level of lipoprotein and a reduced level of adiponectin. Diabetic patients use agents to decrease cardiovascular risk. Hemmati et al. (2015) compared antiatherogenic and antioxidant effects of three medicinal plants B. vulgaris, C. sativus, and Z. Jujuba. Their finding show that saffron, jujube, and barberry extracts might have antiatherogenic effects in diabetic rat models, which is probably to be associated to the antioxidant capacities of the extracts (Hemmati et al., 2015).

\section{Saffron's Effect on Sexual Function}

Reactive oxygen species have impacts on gamete quality and gamete coaction; ROS affect spermatozoa, oocytes, embryos, and their environment. Maleki et al.
(2014) studied the antioxidant effect of different concentrations of saffron aqueous extract and its great component, crocin, on the improvement of in vitro maturation (IVM) and subsequent in vitro fertilization (IVF) and embryo development of mouse oocytes. Using of saffron aqueous extract throughout in vitro maturation can impress on IVM, IVF and early embryo development in a dose dependent manner. Saffron aqueous extract seems to have a stronger effect than pure crocin (Maleki et al., 2014). In folk medicine, saffron is suggested as an aphrodisiac agent. Crocin's aphrodisiac activity was evaluated on male rats. The researches shows that the crocin reveal aphrodisiac activity by increasing mounting frequency (MF), intromission latency (IL) and ejaculation latency (EL) behaviors and reducing MF, IL and EL parameters (Singla and Varadaraj, 2011).

\section{Saffron's Effect on Hyperlipidemia and Hypertension}

Hypertension is the widespread cardiovascular disease and is a main public health disorder in developed as well as developing countries. Saffron contains a chemical called crocetin that lowers the blood pressure (Joshi et al., 2012). Researchers are studied that effects of chronic exposure to saffron stigma aqueous extract on the blood pressure of normotensive and desoxycorticosterone acetate (DOCA)-salt induced hypertensive rats (Imenshahidi et al., 2013). They are concluded that saffron aqueous extract possesses antihypertensive and normalizing effect on blood pressure in chronic application (Imenshahidi et al., 2013). Saffron extract and its components, principally crocin and safranal, have some blood pressure modulating features. But, there must be further more studies to find effective dose and mechanism of action. Saffron's effect can be related to antioxidant properties and its components (Milajerdi et al., 2015).

\section{Anticancer and Antitumor Effect of Saffron}

Cancer sustains to indicate the largest reason of mortality in the world and claims over 6 million lives every year. An excessively preventing strategy for cancer protection today is chemoprevention, which is describes as the use of synthetic or natural agents to stop the development of cancer in human beings. Plants, vegetables, herbs and spices used in folk and traditional medicine have been admitted recently as one of the major sources of cancer chemo preventive drug exploration and development. Chermahini et al. reported that saffron and its components has an inhibitory influence on cellular DNA and RNA synthesis, but not on protein synthesis. It has been shown that nucleic acid synthesis was inhibited by saffron and its constituents without any profound effect on protein synthesis in tumour cells. The antitumor activity of saffron and its components is the inhibitory effect on free radical chain reactions, because most of the carotenoids are soluble in lipid and might act as membrane related high-efficiency free-radical scavengers, which is associated with their antioxidant properties. Another mecanism is that the cytotoxic effect of saffron is connected with interaction of carotenoids with 
topoisomerase II, an enzyme included in cellular DNAprotein interaction (Chermahini et al., 2010). It has been showed that crocin indicates anti-proliferative effects in human colorectal cancer cell lines while not impressing normal cells. In addition to that, researchers report that crocin and crocetin were shown to inhibit breast cancer cell proliferation. Noureini and Wink (2012) investigate that antiproliferative effect of crocin in hepatocarcinoma (HepG2) cells. Telomerase activity of HepG2 cells reduces after treatment with crocin, which is presumably caused by down-regulation of the expression of the catalytic subunit of the enzyme. Studies shows that crocin might be a selective target in cancer therapy (Noureini and Wink, 2012). The antitumor activity of saffron was reported in literature in the beginning of 1990's. Saffron and its carotenoid constituents are chemo preventive in growth human malignant cells and animal models, exhibiting cell growth. This could explain how they reduce outgrowth of tumour cells in vivo. It has been represented that those malignant cells are more susceptible than normal cells against the inhibitory effect of saffron. Inhibition of intracellular nucleic acid synthesis and free radical chain reactions may contribute to describe the molecular mechanism of antitumor effect of saffron (Abdullaev, 2004).

\section{Effects of Saffron in Reperfusion}

There is an investigation on the effects of crocin, a natural antioxidant reproduced from saffron, on cardiac reperfusion-induced arrhythmia and antioxidant systems such as catalase and superoxide dismutase (SOD) enzyme activities, glutathione (GSH) and malondialdehyde (MDA, as a marker of lipid peroxidation) levels (Jahanbakhsh et al., 2012). Their results recommend a preventive role of crocin on cardiac reperfusion arrhythmias which may at least partly be associated to stability or even amplification of antioxidant systems. Crocin might potentially be utilized for treatment or prevention of arrhythmias in patients with ischemic heart disease (Jahanbakhsh et al., 2012).

\section{Conclusions}

Saffron is one of the remarkable plants in the world. Saffron is grown in many countries in worldwide. Many of studies have been investigated about saffron. The studies concentrated in biological activities of saffron.

Since ancient times, saffron has been used as a medicinal plant and a spice. It has been used as an antispasmodic, sedative agent, stimulant, stomachic, reperfusion, ischemia, aphrodisiac, anticonvulsant, antidepressant, anti-inflammatory, antitumor, anticancer, antihypertensive, antioxidant and learning and memory improving effects. It has great effects on central nervous system. The findings report that using traditional medicine saffron is very beneficial to protecting disorders.

People while using saffron, they must be careful because the highest dose of saffron might be toxic. A variety of natural products, including natural extracts and isolated compounds from plants, have been reported to prevent health problems. Herbs are important for prevention from disorders. Antioxidant-rich saffron compounds may modulate disorders.

\section{Competing interest}

The authors declare that they have no conflict of interest.

\section{References}

Abdullaev F. 2007. Biological properties and medicinal use of saffron (Crocus sativus L.) Acta Hort. (ISHS) 739:339-345.

Abdullaev FI. 2004. Antitumor Effect of Saffron (Crocus sativus L.): Overview and Perspectives. ISHS Acta Horticulturae 650: I International Symposium on Saffron Biology and Biotechnology.

Agnihotri VK, Aperito J. 2015. Crocus Sativus Linn: An Informative Review. Aperito Journal of Advanced Plant Biology, 1:103.

Ahmad Dar R, Brahaman PK, Tiwari S, Pitre KS. 2012. Indirect electrochemical analysis of crocin in phytochemical sample. EJournal of Chemistry, 9(2): 918-925.

Ahmad AS, Ansari MA, Ahmad M, Saleem S, Yousurf S, Hoda $\mathrm{MN}$, Islam F. 2005. Neuroprotection by crocetin in a hemiparkinsonian rat model. Pharmacol Biochem. Behav., 81, 805813.

Ahrazem O ${ }^{1}$,Rubio-Moraga A,López RC,Gómez-Gómez L. 2010. The expression of a chromoplast-specific lycopene beta cyclase gene is involved in the high production of saffron's apocarotenoid precursors. Journal of Experimental Botany, Vol. 61, No. 1, pp. 105-119.

Alavizadeh SH, Hosseinzadeh H. 2014. Bioactivity assessment and toxicity of crocin: A comprehensive review. Food and Chemical Toxicology, 64: 65-80.

Arasteh A, Aliyev A, Khamnei S, Delazar A, Mesgari M, Mehmannavaz Y. 2010. Effects of hydromethanolic extract of saffron (Crocus sativus) on serum glucose, insulin and cholesterol levels in healthy male rats. J. Med. Plants Res., 4: $397-402$.

Arslan N, Gürbüz B, İpek A, Özcan S, Sarıhan E, Daeshian AM, Moghadassi MS. 2007. The effect of corm size and different harvesting time on saffron (Crocus sativus L.) regeneration. ISHS Acta Horticulturae 739: II International Symposium on Saffron Biology and Technology.

Bhandari PR. 2015. Crocus sativus L. (saffron) for cancer chemoprevention: A mini review. Journal of Traditional and Complementary Medicine 5: 81-87.

Caiola MG, Canini A. 2010. Looking for saffron's (Crocus sativus L.) parents. Functional Plant Science and Biotechnology 4, Speciel Issue 2: 1-14.

Chen Y, Zhang H, Tian X, Zhao C, Cai L, Liu Y, Jia L, Yin HX, Chen C. 2008. Antioxidant potential of crocins and ethanol extracts of Gardenia jasminoides ELLIS and Crocus sativus L.: A relationship investigation between antioxidant activity and crocin contents. Food Chem., 109: 484 - 92.

Chermahini SH, Abd. Majid FA, Sarmidi MR, Taghizadeh E, Salehnezhad S. 2010. Impact of saffron as an anti-cancer and anti-tumor herb. African Journal of Pharmacy and Pharmacology, Vol 4(11), pp. 834-840.

Davis PH, Mill RR, Tan K. 1988. Flora of Turkey and the east of Aegan Island. 10:245-265.

Hagh-Nazari S, Keifi N. 2007. Saffron and various fraud manners in its production and trades. ISHS Acta Horticulturae 739: II International Symposium on Saffron Biology and Technology.

Hemmati M, Zohoori E, Mehrpour O, Karamian M, Asghari S, Zarban A, Nasouti R. 2015. Anti-Atherogenic potential of jujube, saffron and barberry: Anti-Diabetic and antioxidant actions. EXCLI. Journal, 14:908-915. 
Hosseinzadeh H, Sadeghnia HR, Ghaeni FA, Motamedshariaty VS, Mohajeri SA. 2012. Effects of saffron (Crocus sativus L.) and its active constituent, crocin, on recognition and spatial memory after chronic cerebral hypoperfusion in rats. Phytotherapy Research, 26: 381-386.

Imenshahidi M, Razavi BM, Faal A, Gholampoor A, Mousavi SM, Hosseinzadeh H. 2013. The effect of chronic administration of saffron (Crocus sativus) stigma aqueous extract on systolic blood pressure in rats. Jundishapur J. Nat. Pharm Prod., 8(4): $175-179$.

Jahanbakhsh Z, Rasoulian B, Jafari M, Shekarforoush S, Esmailidehaj M, Mohammadi Mt, Aghai H, Salehi M, Khoshbaten A. 2012. Protective effect of crocin againstiinduced cardiac arrhythmias in anaesthetized arts. EXCLI Journal, 11: 20-29.

Joshi UH, Ganatra TH, Bhalodiya PN, Desai TR, Tirgar PR. 2012. Comparative review on harmless herbs with allopathic remedies as anti-hypertensive. Research Journal of Pharmaceutical, Biological and Chemical Sciences, 3(2): 673-687.

Kamalipour M, Akhondzadeh S. 2011. Cardiovascular effects of saffron: an evidence-based review. FB Pharmacol SCI. J. The. Univ. Heart Ctr., 6(2): 59-61.

Kianbakht S, Hajiaghaee R. 2011. Anti-hyperglycemic effects of saffron and its active constituents, crocin and safranal, in alloxan-induced diabetic rats. Journal of Medicinal Plants, Volume 10: No 39.

Leffingwell JC. 2002. Saffron. Leffingwell Reports, Vol. 2(5).

Makhlouf H, Saksouk M, Habib J, Chahine R. 2011. Determination of antioxidant activity of saffron taken from the flower of Crocus sativus grown in Lebanon. African Journal of Biotechnology, 10: 8093-8100.

Maleki EM, Eimani H, Bigdeli MR, Ebrahimi B, Shahverdi AH, Narenji AG, Abedi R. 2014. A comparative study of saffron aqueous extract and its active ingredient, crocin on the in vitro maturation, in vitro fertilization, and in vitro culture of mouse oocytes. Taiwanese Journal of Obstetrics \& Gynecology 53: 21-25.

Mashmoul M, Azlan A, Khaza'ai H, Mohd Yusof BN, Mohd Noor S. 2013. Saffron: a natural potent antioxidant as a promising anti-obesity drug. Antioxidants, 2: 293-308.

Milajerdi A, Bitarafan V, Mahmoudi M. 2015. A review on the effects of saffron extract and its constituents on factors related to neurologic, cardiovascular and gastrointestinal diseases. Journal of Medicinal Plants, 14(55): 9-28.

Modaghegha MH, Shahabiana M, Esmaeilib HA, Rajbaic O, Hosseinzadeh H. 2008. Safety evaluation of saffron (Crocus sativus) tablets in healthy volunteers. Phytomedicine, 15: 10321037.

Moghadam GD, Sadeghi SM, Droodian H. 2013. Types of cultivation methods in saffron (Crocus sativus) and observing the principles of fight against pests and weeds. Persian Gulf Crop Protection, 2(2): 8-13.

Mohajeri D, Mousavi G, Doustar Y. 2009. Antihyperglycemic and pancrease-protective effects of Crocus sativus L. (saffron) stigma ethanolic extract on rats with alloxan-induced diabetes. J. Biol. Sci., 9: 302 - 10.

Mohajeri D, Mousavi G, Mesgari M, Doustar Y, Nouri MHK. 2007. Subacute toxicity of Crocus Sativus L. (Saffron) stigma ethanolic extract in rats. American Journal of Pharmacology and Toxicology, 2(4): 189-193.

Mohajeri SA, Hosseinzadeh H, Keyhanfar F, Aghamohammadian J. 2010. Extraction of crocin from saffron (Crocus sativus) using molecularly imprinted polymer solid-phase extraction. J. Sep. Sci., 33: 2302-2309.

Mousavi SZ, Bathaie SZ. 2011. Historical uses of saffron: Identifying potential new avenues for modern research. Avicenna Journal of Phytomedicine, 1: 57-66.
Nam KN, Park YM, Jung HJ, Lee JY, Min BD, Park SU, Jung WS, Cho KH, Kang I, hong JW, Lee EH. 2010. Anti-inflammatory effects of crocin and crocetin in rat brain microglinal cells. European Journal of Pharmacology, 648: 110-116.

Noureini SK, Wink M. 2012. Antiproliferative effects of crocin in hepG2 cells by telomerase inhibition and hTERT downregulation. Asian Pacific Journal of Cancer Prevention, 13: 2305-2309.

Ochiai T, Shimeno H, Mishima K, Iwasaki K, Fujiwara M, Tanaka H, Shoyama Y, Toda A, Eyanagi R, Soeda S. 2007. Protective effects of carotenoids from saffron on neuronal injury in vitro and in vivo. Biochim. Biophys. Acta., 1770: 578-584.

Özdemir C, Baran P, Akyol Y. 2006. The morphology and anatomy of Crocus flavus weston subsp. flavus (Iridaceae). Turk J. Bot., 30: $175-180$.

Premkumbar K, Ramesh A. 2010. Anticancer, antimutagenic and antioxidant potential of saffron: An overview of current awareness and future perspectives. Functional plant science and technology, 4: 91-97.

Rahimi A, Rezaee MB, Jaimand K, Ashtiany AN. 2013. The Effects of storage and cultivation condition on crocin content of dried stigma in saffron (Crocus sativus L.) Pure Appl. Bio., 2: 122125.

Sahraeil H, Fatahi Z, Rohani AH, Eidi A, Hooshmandi Z, Tavallaei SA. 2012. Ethanolic extract of saffron and its constituent crocin diminish stress-induced metabolic signs and alterations of dopamine-related behaviours in rats. International Research Journal of Pharmacy and Pharmacology, 2: 165-173.

Sharafzadeh S. 2012. In vitro callus induction in saffron leaves. International Journal of Pharma and Bio Sciences, 3: 172-175.

Sheng L, Qian Z, Shi Y, Yang L, Xi L, Zhao B, Xu X, Ji H. 2008. Crocetin improves the insulin resistance induced by high-fat diet in rats. Br. J. Pharmacol, 154: 1016 - 24.

Singla RK, Varadaraj Bhat G. 2011. Crocin: An Overview. Indo Global Journal of Pharmaceutical Sciences, 1: 281-286.

Tamaddonfard E, Farshid AA, Eghdami K, Samadi F, Erfanparast A. 2013. Comparison of the effects of crocin, safranal and diclofenac on local inflammation and inflammatory pain responses induced by carrageenan in rats. Pharmacological Reports, 65: 1272-1280.

Xi L, Qian Z, Shen X, Wen N, Zhang Y. 2005. Crocetin prevents dexamethasone-induced insulin resistance in rats. Planta Medica, 71: 917 - 22.

Xi L, Qian Z, Xu G, Zhou C, Sun S. 2007. Crocetin attenuates palmitate-induced insulin insensitivity and disordered tumor necrosis factor- $\alpha$ and adiponectin expression in rat adipocytes. Br. J. Pharmacol, 151: $610-7$.

Xiang M, Qian ZY, Zhou CH, Liu J, Li WN. 2006a. Crocetin inhibits leukocyte adherence to vascular endothelial cells induced by AGEs. J. Ethnopharmacol. 107: 25 - 31.

Xiang M, Yang M, Zhou C, Liu J, Li W, Qian Z. 2006b. Crocetin prevents AGEs-induced vascular endothelial cell apoptosis. Pharmacol. Res., 54: 268 - 74.

Yang R, Tan X, Thomas AM, Shen J, Qureshi N, Morrison DC, Van Way III CW. 2006. Crocetin inhibits mRNA expression for tumor necrosis factor alpha, Interleukin- 1beta and inducible nitric oxide synthase in hemorrhagic shock. J. Parenter Enteral Nutr., 30, 297-301.

Zhang Y, Shoyama Y, Sugiura M, Saito H. 1994. Effects of Crocus sativus $\mathrm{L}$. On the ethanol-induced impairment of passive avoidance performances in mice. Biol. Pharm. Bull., 17: 217221.

Zheng YQ, Liu JX, Wang JN, XU L. 2007. Effects of crocin on reperfusion induced oxidative/nitrative injury to cerebral microvessels after global cerebral ischemia. Brain Res., 1138, 86-94. 